\title{
SHORT-TERM EEG PATTERNS OF DRIVER DROWSINESS AND THEIR RELATION TO CRASHES
}

\author{
Martin Golz, David Sommer \\ Faculty of Computer Science, University of Applied Sciences Schmalkalden, Germany \\ m.golz@fh-sm.de
}

\begin{abstract}
Microsleep (MS) and alpha burst (AB) patterns in the EEG of ten young drivers were detected. Their percentage within 1 min intervals was compared with independent variables of drowsiness: 1) lane tracking performance, 2) self-rating of sleepiness. In addition, the occurrence of both patterns immediately before crashes was investigated. Results offer remarkable differences. AB displays no time-since-sleep as well as no time-on-task effect. $A B$ failed in predicting crashes. MS displays both effects and always occurred immediately before crashes.
\end{abstract}

Keywords: EEG, pattern recognition, drowsiness, fatigue, microsleep, driving simulation, support-vector machines

\section{Introduction}

EEG is the most promising signal for reliable estimation of driver drowsiness due to the more or less direct assessment to the origin, the driver's brain. Various authors investigated this topic; mostly, EEG features were extracted followed by correlation analysis or analysis of variance [1 and references therein]. Up to now investigations utilizing short-term pattern recognition account to a minority. Two decades ago, Kecklund and Åkerstedt proposed AB as a marker of drowsiness [2]; recently, this concept was taken up again [3]. Alternatively, recognition of MS utilizing spectral domain, state space features, and kernel classifiers was proposed to quantify drowsiness [4]. We compare both pattern recognition methods based on the same data set and relate their output variables to two independent and broadly accepted variables in drowsiness research.

\section{Methods}

Study: was designed to investigate driving performance and subjects' behaviour under high level of monotony and sleepiness. Monotony was supported by selecting very low traffic density (no car in lane, 1 car every 3 minutes in the opponent lane; road configuration: winding twolane road, undulating landscape). Subjects were instructed to keep in lane as best as possible and to avoid falling asleep. After returning from MS subjects were reminded that if driving performance becomes too bad the experiment would be terminated. Experiments started at 1:00 and ended at 7:40 AM, with time-since-sleep of at least $16 \mathrm{~h}$ and 22:40 h, respectively. Within this time 7 driving sessions (length 40 min long) were carried out.

Driving simulation: were conducted at our lab. Crashes have been defined as intervals where all 4 wheels were out of lane, no matter if the car went to the left or to the right. Incidents with 1 - 3 wheels out of lane were not included in further analysis. Lane tracking performance was measured by the standard deviation of the lateral position in lane (SdLat).

When crashes appeared an extensive soundscape as well as video scene was played to increase the emotional importance of these events. In addition drivers were warned by the observers.

Subjects: 14 healthy young volunteers (age $22.4 \pm 4.1$ years, range 18 - 34) with driving experience for at least 1 year participated. 1 male and 2 female quitted because of simulator sickness, 1 male quitted because of back pain.

Data acquisition: 11 biosignals (EEG: F1, F2, C3, Cz, C4, O1, O2, A1, A2, com. av. ref.; EOG: vertical, horizontal), 3 videos (driver's head \& pose, driver's eyes, driving scene), 2 car related time series (steering angle, lateral position in lane) were recorded. Subjectively experienced sleepiness was rated every 4 min during driving following suggestions of [5]. Subject's response was given orally using the Karolinska Sleepiness Scale (KSS) [6].

Visual Ratings: Two operators who watched the videos performed a first judgment of ongoing MS immediately during the experiments. Typical signs of MS are prolonged eyelid closures, slow roving eye movements, head noddings, slow drifting head movements, and major driving incidents. They were often followed by abrupt reactions. Several other signs were observed, but it has been decided not to solely rely on them. In all, we have found 2,290 MS (per subject: $229 \pm 67$, range $138-363$ ). During off-line scoring an independent and trained rater refined the time of MS occurrence. Visual ratings were only utilized to select examples of MS and Non-MS out of the continuum of the recordings.

MS detection: From EEG / EOG segments (length $6 \mathrm{~s}$ ) of MS and Non-MS log spectral power densities (PSD) were estimated (modified periodogram) and summed in spectral bands $(0.5-23 \mathrm{~Hz}$, width $1 \mathrm{~Hz})$ [4]. In addition delay-vector variances were estimated [7]. Support-Vector Machines (SVM, Gaussian kernel) were trained on these data in order to discriminate MS and Non-MS. After empirical parameter optimizations an MS detector was constructed (accuracy $97.7 \pm 2.1 \%$ ). Next, the MS detector was applied consecutively to all data to detect EEG/EOG patterns which are similar to MS [8].

AB detection: 1 EEG signal (O1) was divided in overlapping segments ( $1 \mathrm{~s}$ length, $75 \%$ overlap) [3]. If PSD culminated within extended alpha range $(7-13 \mathrm{~Hz})$ then the full width at half maximum (FWHM) was checked to be lower than two times the bandwidth of the Hamming window applied for spectral estimation. A polynomial model was fitted to the actual and to the mean PSD spectrum. Signal energy as the area above and noise energy as the 
area below the fitted curve can be separated which is needed for amplitude normalization to compensate varying noise levels. Only EEG segments having signal energy within the FWHM of at least twice as large as the noise energy and having a relative peak frequency shift lower than $10 \%$ were considered as AB [3] (Tab. 1).

Table 1: Comparison of both pattern recognition methods.

\begin{tabular}{lcc}
\hline & AB detection & MS detection \\
\hline processed signals & $1(\mathrm{EEG})$ & $9(\mathrm{EEG})+2(\mathrm{EOG})$ \\
temporal resolution & high $(0.25 \mathrm{~s})$ & high $(0.1 \mathrm{~s})$ \\
pattern duration & $0.5-3.0 \mathrm{~s}$ & $1.0-12.0 \mathrm{~s}$ \\
pattern characterization & in spectral domain & in spectral domain \\
pattern definition & fixed & adaptive \\
pattern recognition & fixed decision rules & nonlinear discrimi- \\
method & with thresholds & nant functions \\
signal/noise separation & exponential fit & none \\
output variable & pattern percentage per interval \\
\hline
\end{tabular}

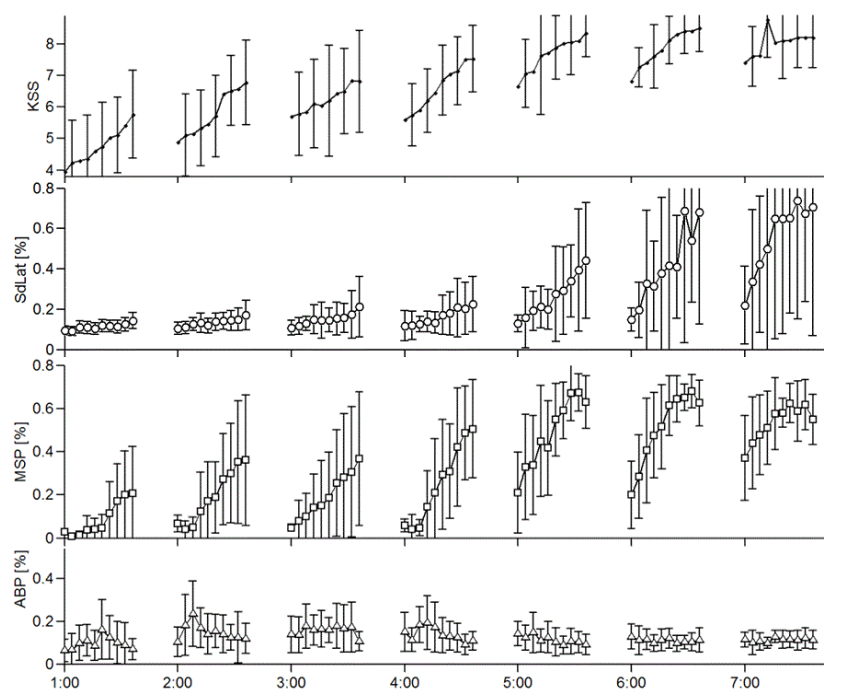

Figure 1: Mean and standard deviation of self-ratings of sleepiness on KSS, lane tracking performance SdLat, MS, and $\mathrm{AB}$ percentage versus time of day

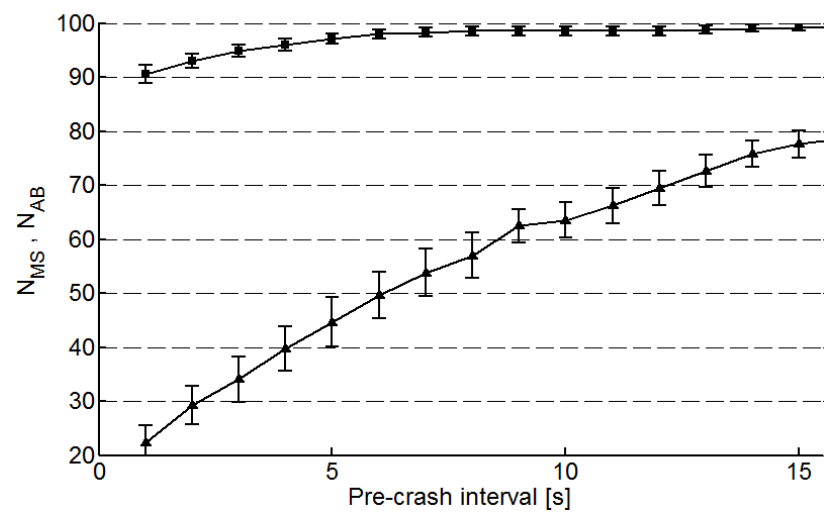

Figure 2: Mean and standard errors of pre-crash intervals containing detections of MS and $\mathrm{AB}$ (lower curve)

\section{Results}

Mean values of KSS, SdLat, and MS percentage correlate with time-since-sleep as well as with time-on-task, whereas $\mathrm{AB}$ percentage remained unchanged across the night and within driving sessions (Fig. 1). AB percentage dis- plays larger standard deviation during the first 4 driving sessions and lower variability during the last sessions where subjects report on high self-experienced sleepiness and perform worse with regard to the lane tracking performance SdLat.

For each crash it was investigated if at least one MS and one $\mathrm{AB}$ pattern was detected within pre-crash intervals of varying length (Fig. 2). The relative number of pre-crash intervals containing MS patterns $N_{M S}$ was always above $90 \%$, but not so with AB patterns. There were many crashes where no $\mathrm{AB}$ was detected immediately before.

\section{Discussion}

This comparative investigation of two EEG pattern recognition methodologies found remarkable differences. AB pattern appeared relatively often and with low duration. They had no significant time on task as well as no time since sleep effect. This is in contrast to the temporal development of behavioral signs of drowsiness as well as with both independent variables of drowsiness. The increase of $\mathrm{AB}$ percentage within the first half of the night might be interpreted as early indications of sleepiness. During the second half of the night where driver's performance dramatically deteriorated $\mathrm{AB}$ remained insensitive. Moreover, the results of crash analysis showed that $\mathrm{AB}$ seemed to arise spontaneously and not strictly related to degradations due to drowsiness. This is in contrast to [3] where an increase of $\mathrm{AB}$ rate from $7 \mathrm{~min}^{-1}$ up to 12.8 $\mathrm{min}^{-1}$ between the first and the last $20 \mathrm{~min}$ of driving was registered. An increase of $\mathrm{AB}$ duration from $0.62 \mathrm{~s}$ up to $0.665 \mathrm{~s}$ was reported which we could not verify.

In contrast, MS patterns turned out to be reliable forerunners of crashes. In more than $95 \%$ of all crashes MS were found immediately before. The MS concept has the potential to establish a gold standard of drowsiness evaluation.

\section{Bibliography}

[1] Khushaba, R. et al.: Driver drowsiness classification using fuzzy-wavelet-based feature extraction, IEEE Trans. Biomed. Eng., vol. 58, pp. 121-131, 2011

[2] Kecklund, G., Åkerstedt, T.: Sleepiness in long distance truck driving, Ergonomics, vol. 36, pp. 1007-1017, 1993

[3] Simon, M., Schmidt, E. et al.: EEG alpha spindle measures as indicators of driver fatigue under real traffic, $J$ Clin. Neurophysiol., vol. 122, pp. 1168-1178, 2011

[4] Golz, M., Sommer, D. et al.: Feature fusion for the detection of microsleep events, J VLSI Signal Proc. Syst., vol. 49, pp. 329-342, 2007

[5] Ingre, M., Åkerstedt, T. et al.: Subjective sleepiness, simulated driving performance and blink duration. $J$. Sleep Res., vol. 15, pp. 47-53, 2006

[6] Åkerstedt, T.: Subjective \& objective sleepiness in the active individual. Int $J$ Neurosci, vol. 52, pp. 29-37, 1990

[7] Gautama, T., Mandic, D., Van Hulle, M.: Novel method for determining the nature of time series, IEEE Trans. Biomed. Eng., vol. 51, pp. 728-736, 2004

[8] Golz, M., Sommer, D. et al.: Microsleep episodes and related crashes during overnight driving simulations. Proc. $6^{\text {th }}$ Int. Sympos. Driver Assess., pp. 39-45, 2009 